\title{
Mutation of luxS affects motility and infectivity of Helicobacter pylori in gastric mucosa of a Mongolian gerbil model
}

\author{
Takako Osaki, ${ }^{1}$ Tomoko Hanawa, ${ }^{1}$ Taki Manzoku, ${ }^{1}$ Minoru Fukuda, ${ }^{2}$ \\ Hayato Kawakami, ${ }^{3}$ Hidekazu Suzuki, ${ }^{4}$ Hiroyuki Yamaguchi, ${ }^{5}$ Xu Yan, ${ }^{6}$ \\ Haruhiko Taguchi, ${ }^{1}$ Satoshi Kurata ${ }^{1}$ and Shigeru Kamiya ${ }^{1}$ \\ 1,2,3 Department of Infectious Diseases ${ }^{1}$, Laboratory for Electron Microscopy ${ }^{2}$ and Second \\ Department of Anatomy ${ }^{3}$, Kyorin University School of Medicine, Shinkawa, Mitaka, Tokyo, \\ Japan \\ ${ }^{4}$ Department of Internal Medicine, Keio University School of Medicine Shinjuku-ku, Tokyo, \\ Japan \\ ${ }^{5}$ Department of Basic Laboratory Sciences, School of Allied Health Sciences, Osaka \\ University, Yamadaoka, Suita, Osaka, Japan \\ ${ }^{6}$ Department of Microbiology, Kunming Medical School, Kunming, Republic of China
}

Correspondence

Takako Osaki

osaki@kyorin-u.ac.jp

Received 4 April 2006

Accepted 18 July 2006
Helicobacter pylori is associated with gastric disorders in humans and some experimental animals, and possesses the luxS/type 2 autoinducer (Al-2) system. The effects of a specific luxS mutation on the characteristics of $H$. pylori were examined. On $0.3 \%$ agar medium, motility of H. pylori HPKY08 (luxS : : cat) was significantly lower than that of wild-type H. pylori TK1402. The luxS-complemented strain HPKY21 exhibited motility comparable to that of $H$. pylori TK1402. It was shown that the luxS/Al-2 system plays an important role in $H$. pylori motility. The luxS mutant exhibited a reduced infection rate relative to the wild-type parent strain TK1402 in a Mongolian gerbil model. At 3 months after oral inoculation, lower numbers of $H$. pylori were detected by semi-quantitative real-time reverse transcription PCR (qRT-PCR) in lux $S^{-}$ mutant-infected gerbils than in TK1402-infected gerbils. Gastric inflammation and increased antibody titre for $H$. pylori were observed in TK1402-infected gerbils only.

\section{INTRODUCTION}

Quorum sensing (QS) is a cell-to-cell communication system that regulates bacterial phenotypes, including the expression of virulence factor genes. The signalling molecules are known as autoinducers (AIs), and when these molecules reach a critical threshold concentration within a bacterial population, a signal transduction cascade is triggered, and this forms the basis for alterations in gene expression (Fuqua et al., 1994). Many Gram-negative bacteria utilize $\mathrm{N}$-acylhomoserine lactone molecules, AI-1, as signals, while Gram-positive bacteria actively export peptides as signalling molecules. There is a second signalling system involved in a wide range of bacterial species (Schauder et al., 2001), and this system is utilized by both Gram-positive and Gram-negative bacteria. The signalling molecule known as type 2 autoinducer (AI-2) is a furanosyl borate diester (Chen et al., 2002), and the enzyme responsible for its synthesis is encoded by the luxS gene (Surette \&

Abbreviations: Al-2, type 2 autoinducer; qRT-PCR, semi-quantitative real-time reverse transcription PCR.
Bassler, 1999). The genomes of many bacterial species, notably Escherichia coli, Salmonella enterica serovar Typhimurium, Shigella flexneri, Proteus mirabilis, Vibrio cholerae, Vibrio vulnificus, Campylobacter jejuni, Porphyromonas gingivalis, Bacillus subtilis, Streptococcus pyogenes, Streptococcus mutans, Clostridium perfringens and Clostridium difficile, include luxS homologues. In several of these, luxS-related AI-2 signals are involved in bacterial characteristics such as biofilm formation (Balestrino et al., 2005; Blehert et al., 2003; Fong et al., 2001; Wen \& Burne 2004), flagella and motility (Jeon et al., 2003; Schneider et al., 2002; Stroeher et al., 2003), type III secretion systems (Sperandio et al., 1999), toxin production (Ohtani et al., 2002) and virulence (Lyon et al., 2001; Parsonnet et al., 1991; Stroeher et al., 2003).

Helicobacter pylori has been identified as the aetiological agent of chronic active gastritis, peptic ulcer disease (Blaser, 1992; Graham, 1989), gastric adenocarcinoma (Parsonnet et al., 1991) and mucosal-associated lymphoid tissue (MALT) lymphoma (Wotherspoon et al., 1993). H. pylori has flagellar motility and is able to move through the 
viscous mucus gel lining the epithelium of the human stomach, thus leading to chronic infection of the human gastric mucosa. $H$. pylori produces extracellular signalling molecules related to AI-2, and production of AI-2 is dependent on luxS function (Forsyth \& Cover, 2000; Joyce et al., 2000). These authors have reported that the production of AI-2 by luxS is growth-phase dependent, with maximal production occurring in the mid-exponential phase of growth. Furthermore, in their studies, maximal accumulation of AI-2 in the culture occurred in stationary phase. It has also been reported that the expression of $H$. pylori flaA is growth-phase dependent (Thompson et al., 2003), and that flaA transcription increases with culture density (Loh et al., 2004) at stationary phase. Mutating the luxS gene eliminates the growth-phase dependence of flaA, and growth-phase dependence is restored when the luxS mutant is complemented with wild-type luxS. Accordingly, we expected that the mutation of luxS of $H$. pylori might affect motility in the stationary phase, but not in exponential phase.

Several experimental animal models, such as monkeys (Drazek et al., 1994; Fujioka et al., 1997; Schauder et al., 2001), gnotobiotic piglets (Eaton et al., 1997), C57/BL6 mice (Lee et al., 1997; Kim et al., 2003) and Mongolian gerbils, are helpful in understanding the pathogenesis of human $H$. pylori infection. The Mongolian gerbil model, in which $H$. pylori is able to colonize long-term, is particularly easy to handle, and is useful as a small-animal model for the severe inflammation and obvious ulceration caused by $H$. pylori (Hirayama et al., 1996; Matsumoto et al., 1997; Yokota et al., 1991). The aim of this study is to investigate the effects of a defined luxS mutation on the characteristics of $H$. pylori and to determine whether the absence of AI-2 production allows $H$. pylori to infect and/or maintain infection in the gerbil gastric mucosa.

\section{METHODS}

Strains and culture. H. pylori strain TK1402 was isolated from a gastric ulcer and duodenal ulcer patient. This strain exhibits infectivity in germ-free mice (Osaki et al., 1998), C57/BL6 mice (Yamaguchi et al., 2000) and Mongolian gerbils (Nakagawa et al., 2005). In addition, TK1402 is cagA positive and produces vacuolating cytotoxin (VacA). H. pylori TK1402 and its mutant strains were cultured under microaerobic conditions at $37^{\circ} \mathrm{C}$ either on a brain heart infusion agar plate containing $7 \%$ horse blood or in Brucella broth containing $7 \%$ horse serum (Brucella-serum broth). The reporter strains Vibrio harveyi BB170 (luxN::Tn5) and BB152 (luxL::Tn5) were kindly provided by Dr B. L. Bassler (Princeton University). V. harveyi strains were routinely cultured at $30^{\circ} \mathrm{C}$ on Luria-marine (LM) agar.

Construction of $\boldsymbol{H}$. pylori luxS mutant. Primers HP105F2 (5'GCTATTGCCTTGCAACAAATCCCCGC- $\left.3^{\prime}\right)$ and HP105R2 (5'ATACTTAGGCGGGCATAGCGATG- $3^{\prime}$ ) were used to PCR-amplify a $1752 \mathrm{bp}$ fragment containing the $H$. pylori luxS gene allele (luxS: : cat) with genomic DNA from strain TN2 (luxS: : cat) as template. The primers and genomic DNA of TN2 were kindly provided by Drs Ogura and Berg (Washington University, St Louis, MO). The fragment was then introduced directly into $H$. pylori 1402 by natural transformation via allelic exchange, and chloramphenicol-resistant colonies were isolated. Gene disruption in the mutant (designated HPKY08) was confirmed by PCR using the primer pairs HP105F2 and HP105R2, and HP02F (5'-CATACGAAAAAAGCCGCCC-3') and HP02R ( $5^{\prime}$-CTTGTCTGTGGGGATTGAG-3'), which yielded a PCR product of increased size. The mutation was also confirmed by Southern blot analysis with a probe containing the $\operatorname{luxS}$ gene. The mutant HPKY08 was used in all subsequent experiments.

Complementation of IuxS mutant. The gene luxS along with its promoter region was amplified using primers 105F2 and 105R2. This fragment was then cloned into the SspI site adjacent to the cat gene in pGEM. The resultant plasmid, pTKY451, was then transformed into the $\operatorname{luxS} S^{-}$strain by natural transformation and was selected on Brucella-serum agar containing $5 \mu \mathrm{g}$ kanamycin $\mathrm{ml}^{-1}$, and strain HpKY21, with luxS inserted in the chromosome, was obtained. Mutation of $\operatorname{luxS}$ and restoration of $\operatorname{luxS}$ function in the mutant were confirmed by AI-2 assay and Southern blot analysis of genomic DNA digested with SspI, using probes for the luxS gene, the chloramphenicol acetyl transferase gene (cat) and the kanamycin-resistance gene (aph) (Fig. 1).

Al-2 assay. In order to confirm the production of AI-2 by $H$. pylori TK1402, V. harveyi AI-2 reporter strain BB170, which lacks the AI-1 sensor but contains an intact sensor for AI-2, was used in a bioluminescence assay (Table 1$). V$. harveyi was grown at $30^{\circ} \mathrm{C}$ in autoinducer bioassay (AB) broth medium (Hardie et al., 2003). Luminescence bioassay was performed as described previously (Forsyth \& Cover, 2000). H. pylori or V. harveyi BB152 was grown in Brucella-serum broth, and culture supernatants were collected at various time points. Cell-free conditioned medium (CM) was prepared by centrifuging $H$. pylori or $V$. harveyi BB152 cultures in Brucella-serum broth at $8000 \mathrm{~g}$ for $15 \mathrm{~min}$, followed by filtration (pore size $0 \cdot 2 \mu \mathrm{m}$ ). CM preparations were routinely stored at $-30{ }^{\circ} \mathrm{C}$ until use. The $18 \mathrm{~h}$ culture of $V$. harveyi $\mathrm{BB} 170$ was diluted 1:5000 in fresh $\mathrm{AB}$ medium. The experimental $\mathrm{CM}$ preparation

(a)

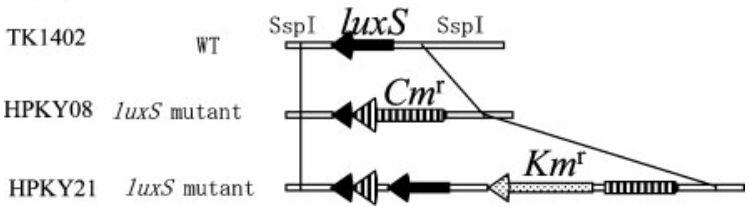

(b)

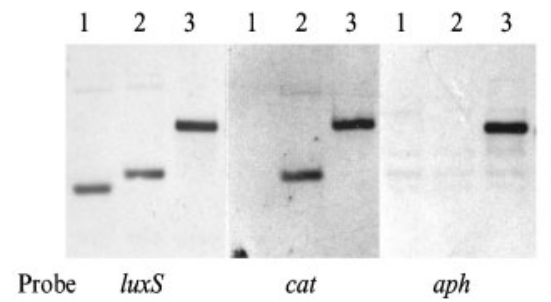

Fig. 1. (a) Construction of luxS mutant strain (HPKY08) and mutant complemented with luxS gene (HPKY21). WT, wildtype. (b) Southern blot analysis of wild-type TK1402 strain (lane 1) and its mutant strains (lane 2, HPKY08; lane 3, HPKY21), using luxS, cat and aph genes as probes. 
Table 1. Al-2 production by $H$. pylori strains

All strains were incubated in Brucella-serum broth.

\begin{tabular}{|c|c|c|c|c|}
\hline Strains & Characteristics & $\begin{array}{c}\text { Time } \\
(\mathbf{h})\end{array}$ & $\begin{array}{c}\text { Number of } \\
\text { H. pylori [log (c.f.u. } \\
\left.\left.\mathrm{ml}^{-1}\right)\right]\end{array}$ & $\begin{array}{c}\text { Activation of } \\
\text { luminescence in } \\
\text { V. harveyi } \mathrm{BB} 170(\%)^{*}\end{array}$ \\
\hline \multicolumn{5}{|l|}{ H. pylori } \\
\hline \multirow[t]{3}{*}{ HРКY08 } & $\operatorname{luxS}:$ : cat & 24 & $10^{8 \cdot 78}$ & $0 \cdot 4$ \\
\hline & & 33 & $10^{8 \cdot 74}$ & $1 \cdot 6$ \\
\hline & & 54 & $10^{8 \cdot 56}$ & $2 \cdot 6$ \\
\hline \multirow[t]{3}{*}{ TK1402 } & Wild-type, clinical isolate & 24 & $10^{8 \cdot 60}$ & $109 \cdot 9$ \\
\hline & & 33 & $10^{9 \cdot 15}$ & $326 \cdot 5$ \\
\hline & & 54 & $10^{9 \cdot 15}$ & $69 \cdot 1$ \\
\hline HPKY021 & luxS complemented & 33 & $10^{9 \cdot 36}$ & $94 \cdot 3$ \\
\hline \multicolumn{5}{|l|}{ V. harveyi } \\
\hline BB152 & $\operatorname{luxL}:: \operatorname{Tn} 5$ & 18 & - & 100 \\
\hline
\end{tabular}

${ }^{*}$ At various time points, total luminescence was quantified. The luminescence of the culture supernatant of V. harveyi BB152 was taken as $100 \%$.

(15 $\mu \mathrm{l})$ was added to the diluted $V$. harveyi culture $(135 \mu \mathrm{l})$, which was then incubated at $30^{\circ} \mathrm{C}$ with agitation (180 r.p.m.). Next, $100 \mu \mathrm{l}$ of this culture was added to a 96-well plate at various time points, and total luminescence was quantified using a luminometer (Mithras LB940, Berthold Technologies). Viable cell counts of $V$. harveyi $\mathrm{BB} 170$ were determined by culture using LM agar, and relative light units were calculated.

Motility assay. Motility of $H$. pylori was assessed on $0.3 \%$ agar Brucella medium containing $7 \%$ horse serum. H. pylori strains grown in Brucella-serum broth for $24 \mathrm{~h}\left(\mathrm{OD}_{600} 0 \cdot 6\right)$ were collected and centrifuged in $1.5 \mathrm{ml}$ tubes. Pellets were inoculated into medium using a sterile picker. Several days after incubation under microaerobic conditions at $37^{\circ} \mathrm{C}$, the diameter of the halo was measured. At least three plates were used for each experiment. The mean diameter was analysed statistically.

The velocity of each $H$. pylori strain in Brucella broth containing $0.5 \%$ glucose was also assessed using video systems. $H$. pylori was irradiated by the slanted dark-field light of a phase-difference condenser (c-c ph condenser, Nikon) and by observing scattered rays; $H$. pylori was detected as a bright point, which was not distinct in liquid medium against a light field. The obtained dark-field images were photographed at 30 frames per second sequentially by a 3CCD camera (DC-330, Dage-MTI), and transferred to computer in AVI file protocol through a capture card (SIM-PCI, DITECT). Motility was analysed using motility analysis software (DIPP-MOTION2D, DITECT). The movement of $H$. pylori on every frame was tracked for $1 \mathrm{~s}$, and each coordinate was determined. The velocity of each $H$. pylori cell was calculated by the same software from coordinates using a frequency distribution chart. In one sample, $60 \mathrm{H}$. pylori bodies were tracked, and the mean velocities of wild-type and mutant strains were estimated and compared.

Ultrastructural study. For scanning electron microscopy, cultures of $H$. pylori strains in Brucella-serum broth were collected and attached to poly-L-lysine-coated cover slips (IWAKI) by centrifugation $(1500 \mathrm{~g}, 5 \mathrm{~min})$. Cells on the cover slips were washed twice in PBS and fixed with $2 \%$ glutaraldehyde. Specimens were examined using a scanning electron microscope (JEOL JSM-5600LV).

Acid tolerance. Brucella broth supplemented with $10 \%$ fetal calf serum was adjusted to $\mathrm{pH} 3$ or $\mathrm{pH} 1$ with $\mathrm{HCl}$, and precipitated proteins were removed by filtration [acid-precipitated (AP) Brucella broth]. Next, $1-3 \times 10^{6}$ c.f.u. H. pylori $\mathrm{ml}^{-1}$ was inoculated into AP Brucella broth, with or without $30 \mathrm{mM}$ urea, at $\mathrm{pH} 1$ or 3 . After 1 or $3 \mathrm{~h}$ incubation at $37^{\circ} \mathrm{C}$, viable bacterial counts were determined.

Animal experiments. Mongolian gerbils (MGS/sea; age 8 weeks; male) purchased from Seac Yoshitomi were fasted for 2 days and inoculated per os with $1-2 \times 10^{9}$ c.f.u. H. pylori TK1402 or HPKY08 (luxS $S^{-}$mutant) in Hanks' solution (Sigma) on two consecutive days. At each time point, four to five gerbils per group were sacrificed, and one half of the stomach was fixed in Carnoa's solution for histological analysis and the mucus layer of the other half was scraped off with a spatula and collected into $1 \mathrm{ml}$ Hanks' solution. Of this $1 \mathrm{ml}$, $0 \cdot 1 \mathrm{ml}$ was inoculated onto M-BHM medium (Nikken Bio Medical Laboratory) and cultured at $37^{\circ} \mathrm{C}$ for 7 days under microaerobic conditions. After the incubation period, gold colonies that exhibited urease activity were counted. Another $0 \cdot 1 \mathrm{ml}$ was treated with RNA protect (Qiagen) and stored at $-80 \mathrm{C}$ until used for RNA preparation.

The infective rate was determined by semi-quantitative real-time reverse transcription PCR (qRT-PCR), which detects expression of $16 \mathrm{~S}$ rRNA (Nakagawa et al., 2005). Total RNA was extracted from samples by RNeasy Mini kit (Qiagen), according to the manufacturer's instructions. Contaminating chromosomal DNA was digested with DNase I (DNA-free, Ambion) $\left[1 \mathrm{U}(\mu \mathrm{g} R \mathrm{RN})^{-1}\right]$ for $20 \mathrm{~min}$ at $37^{\circ} \mathrm{C}$. One microgram of DNase I-treated total RNA was used with avian myeloblastosis virus (AMV) reverse transcriptase and random primers in a commercial reaction mixture $(20 \mu \mathrm{l}$; AMV Reverse Transcription System, Promega) for first-strand complementary DNA synthesis.

Quantitative analysis was performed using the SYBR Green method. The generation of quantitative data was based on the different PCR kinetics of samples with various levels of target-gene expression. cDNA was amplified using PCR primers for $H$. pylori 16S rRNA, 16S2-F (5'-CGCTAAGAGATCAGCCTATGTCC-3') and 16SB2-R (5'-CCGTGTCTCAGTTCCAGTGTGT-3'). Amplification of the glyceraldehyde-3-phosphate dehydrogenase (G3PDH) gene on cDNA derived from Mongolian gerbils using the primers G3PDH-F $\left(5^{\prime}\right.$-ACCACAGTCCATGCCATCAC-3') and G3PDH-R (5'-TCCAC- 
CACCCTGTTGCTGTA-3') (RT-PCR Primer Set, TOYOBO) was used as a control and for standardization of target gene transcriptional activity data. For each primer set, PCR was performed in triplicate. Quantitative data were calculated from a standard curve generated by amplifying serial dilutions of a known quantity of amplicon. For this approach, the specificity of the PCR product was confirmed by dissociation curve analysis (7500 quantification program, Applied Biosystems).

ELISA. Serum antibodies from gerbils infected with $H$. pylori were assessed by ELISA at 3 months after infection, as previously reported (Nakagawa et al., 2005). A 96-well plate was coated with sonicated H. pylori TK1402 antigen ( $3 \mu \mathrm{g}$ per well). Each serum sample was diluted 300-fold with PBS and added to the plates. Bound immunogloblins were detected with protein G-conjugated horseradish peroxidase (Sigma) and were developed using $0 \cdot 1 \% o$-phenylenediamine and $0.035 \% \mathrm{H}_{2} \mathrm{O}_{2}$ in developing buffer $(0 \cdot 1 \mathrm{M}$ citric acid, $0 \cdot 07 \mathrm{M}$ sodium phosphate dibasic). After incubation at room temperature for $5 \mathrm{~min}$, the reaction was stopped by adding $50 \mu \mathrm{l} 2\left(\mathrm{NH}_{2}\right) \mathrm{SO}_{4}$. $A_{490}$ was measured using a microplate reader (model 550, Bio-Rad).

Cell culture and characterization of $\boldsymbol{H}$. pylori. Adhesion activity of $H$. pylori strains was assessed by flow cytometry, as previously reported (Osaki et al., 1997). AGS cells, originating from a gastric adenocarcinoma cell, were cultured in RPMI 1640 medium containing $10 \%$ fetal calf serum under $5 \% \mathrm{CO}_{2}$ at $37^{\circ} \mathrm{C}$. Cells $\left(5 \times 10^{5}\right)$ were cultured with PKH-2-labelled HPKY08 (luxS $S^{-}$mutant) or the wild-type strain for $1 \mathrm{~h}$ at room temperature. The fluorescence intensity of the cells, which reflects adhesion activity, was analysed by flow cytometry (FACSVantage, Becton Dickinson).

Statistical analysis. Statistically significant differences were examined by Student's or Welch's $t$ test.

\section{RESULTS AND DISCUSSION}

\section{Al-2 production by luxS ${ }^{-}$mutant}

It was shown that AI-2 production by wild-type TK1402 was maximal at $33 \mathrm{~h}$ after inoculation, after which it gradually decreased (Table 1). At 24, 33 and $54 \mathrm{~h}$ after inoculation, a cell-free culture of HPKY08 (luxS $S^{-}$mutant) did not yield bioluminescence in the assay, indicating no significant production of AI-2. However, cell-free culture of luxScomplemented HPKY21 showed AI-2 activity. These results clearly indicate that the luxS gene is responsible for the production of AI-2 in $\mathrm{H}$. pylori. These data were in agreement with the results obtained by Forsyth \& Cover (2000) and Joyce et al. (2000). In other words, H. pylori luxS plays an essential role in the production of extracellular signalling molecules.

\section{Growth of luxS mutant}

The microaerobic growth of HPKY08 (luxS $S^{-}$mutant) in Brucella-serum broth medium was compared to that of TK1402. In the exponential phase $(0-16 \mathrm{~h})$, the generation times for TK1402 and HPKY08 were $3 \cdot 1 \pm 0 \cdot 3 \mathrm{~h}$ and $4 \cdot 0 \pm 0 \cdot 8 \mathrm{~h}$, respectively $(P=0 \cdot 1789, n=3)$. From the late-exponential to the stationary phase $(24-64 \mathrm{~h})$, the mean bacterial number $\left(10^{7 \cdot 65} \pm 10^{0.82}\right.$ c.f.u. $\left.\mathrm{ml}^{-1}\right)$ for HPKY08 was slightly lower than that for TK1402 $\left(10^{8 \cdot 33} \pm 10^{0 \cdot 68}\right.$ c.f.u. $\left.\mathrm{ml}^{-1}\right) \quad(P=0 \cdot 1035, \quad n=3) . \quad$ These results indicate a slight difference in growth characteristics between TK1402 and HPKY08 during the late-exponential phase. The generation time and mean bacterial number for HPKY21 complemented with luxS were $3 \cdot 3 \pm 0 \cdot 2 \mathrm{~h}$ and $10^{8 \cdot 23} \pm 10^{0 \cdot 81}$ c.f.u. $\mathrm{ml}^{-1}$, respectively, which were not significantly different from those of the TK1402 strain $(P=0 \cdot 6617$ and $0 \cdot 7886$, respectively). These results suggest that the growth delay in $\mathrm{H}$. pylori may be due to the effects of luxS mutation.

\section{Motility on semi-solid agar and flagella formation of the HPKY08 (luxS ${ }^{-}$mutant) strain}

Three strains of $H$. pylori were cultured on Brucella-serum medium containing $0 \cdot 3 \%$ agar. After 5 days of culture, halo diameters were measured. The HPKY08 (luxS ${ }^{-}$mutant) was motile, but the halo diameter $(8 \cdot 0 \pm 1 \cdot 4 \mathrm{~mm} ; n=4)$ after 5 days of culture was significantly smaller than that of TK1402 $(12 \cdot 3 \pm 2 \cdot 0 \mathrm{~mm} ; n=8)(P<0 \cdot 05) \quad$ (Fig. 2). The mean halo diameter of HPKY21 complemented with luxS was $14 \cdot 0 \pm 1 \cdot 2 \mathrm{~mm}(n=4)$, which was not significantly different from that of TK1402. These results confirmed that the reduction in $H$. pylori motility may be due to the effects of luxS mutation.

In both the wild-type and $\operatorname{luxS} S^{-}$strains, three to five polar flagella were observed by electron microscopy after $15 \mathrm{~h}$ incubation (Fig. 3). No morphological difference was observed between the two strains harvested after 15, 24 and $48 \mathrm{~h}$ cultivation.

On the $0 \cdot 3 \%$ agar-containing medium, the motility of H. pylori HPKY08 (luxS: : cat) was significantly lower than that of the wild-type TK1402 strain. In contrast, the luxScomplemented strain HPKY21 exhibited motility comparable to that of $H$. pylori TK1402. These results suggest that the luxS/AI-2 system plays an important role in $H$. pylori motility. There have been similar reports in other bacteria. Jeon et al. (2003) have reported that mutation of luxS in C. jejuni reduces the transcription of flaA and results in

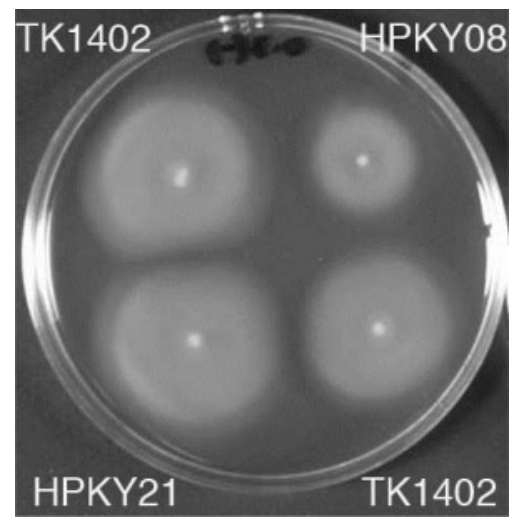

Fig. 2. Motility of HPKY08 (luxS mutant), HPKY21 (luxS complemented) and TK1402 (wild-type) strains on Brucella-serum semi-solid agar after 5 days culture. 


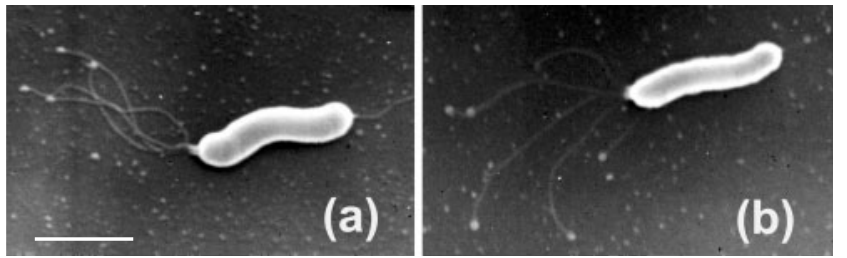

Fig. 3. Flagella formation of HPKY08 (a) and TK1402 (b). Both strains were inoculated in Brucella-serum broth, and the morphology of the strains was analysed by scanning electron microscopy after $15 \mathrm{~h}$ incubation. Bar, $2 \mu \mathrm{m}$.

reduced motility. In the luxS mutant of enteropathogenic E. coli (EPEC), less flagellin is detected on Western blotting, and motility is decreased on semi-solid agar (Sircili et al., 2004). The present study is the first to show significantly reduced motility of the $H$. pylori luxS mutant. Recently, it has been shown that mutating the $H$. pylori luxS gene eliminates growth-phase-dependent control of flaA (Loh et al., 2004).

\section{Effects of glucose on $\boldsymbol{H}$. pylori motility}

To evaluate the influence of glucose on the motility of $H$. pylori, HPKY08 (luxS $S^{-}$mutant) was cultured on Brucella semi-solid medium containing various concentrations of glucose (Fig. 4). On the medium containing 0.13-2\% glucose, HPKY08 motility was significantly inhibited. On the other hand, the motility of both wild-type and luxScomplemented strains was enhanced on medium containing $0 \cdot 13-1 \%$ glucose. At each concentration from 0 to $2 \%$ glucose, the motility of the mutant was significantly lower

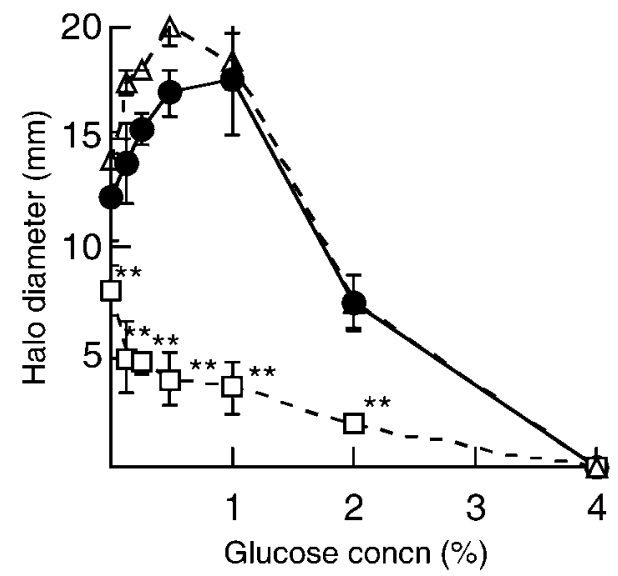

Fig. 4. Effect of glucose on motility of HPKY08 (/uxS mutant; $\square$, dashed line), HPKY21 (luxS complemented; $\triangle$, dashed line) and TK1402 (wild-type; $\bullet$, solid line) strains. The strains were cultured on Brucella-serum semi-solid agar for 4 days, and the diameter of the halo was measured. ${ }^{*}$ The mean diameter of halo was significantly lower than that of TK1402 $(P<0.01)$. than that of the wild-type and luxS-complemented strains $(P<0 \cdot 05)$ (Fig. 4). These results indicate that the motility of the luxS mutant is more sensitive to glucose.

To confirm the inhibitory effect of glucose on the motility of $H$. pylori luxS $S^{-}$mutant strains, the velocity of $H$. pylori strains was determined by a video system. In Brucella medium containing $0.5 \%$ glucose and $7 \%$ horse serum, the mean velocity of $H$. pylori strain HPKY08 $\left(\mathrm{OD}_{600} 0 \cdot 9\right)$ was $6 \cdot 57 \pm 2.62 \mu \mathrm{m} \mathrm{s}^{-1}$, significantly slower than that of the wild-type $\left(12 \cdot 02 \pm 3 \cdot 88 \mu \mathrm{m} \mathrm{s}^{-1} ; \mathrm{OD}_{600} 1 \cdot 2\right)$ and luxScomplemented $\left(10 \cdot 32 \pm 3 \cdot 20 \mu \mathrm{m} \mathrm{s}^{-1} ; \mathrm{OD}_{600} 1 \cdot 0\right)$ strains $(P<0 \cdot 05)$ at $20 \mathrm{~h}$ after inoculation. These results indicate that the reduced motility in $\mathrm{H}$. pylori in the early stationary phase of culture was due to the effects of luxS mutation.

We demonstrated that the presence of glucose $(0 \cdot 13-2 \%)$ strongly inhibited the motility of the luxS $S^{-}$mutant strain, although its growth was not inhibited by glucose $(0 \cdot 13-1 \%)$ (data not shown). Kim et al. (2003) have reported that $V$. vulnificus shows increased signalling activity when cultured in the presence of $0.5 \%$ glucose without growth enhancement. It has been reported that AI-2 production by virulent E. coli and S. Typhimurium is affected by glucose and $\mathrm{pH}$ level (Surette \& Bassler, 1999). Similarly, in the present study, AI-2 production by the $H$. pylori wild-type TK1402 strain was significantly enhanced in the presence of $0.5 \%$ glucose (data not shown). We also checked directly the movement of $H$. pylori strains in the Brucella-broth medium containing glucose, and showed that the velocity of the $H$. pylori luxS mutant strain was significantly lower than that of the wild-type and luxS-complemented strains.

\section{Acid tolerance of the HPKY08 strain}

Both wild-type and HPKY08 strains were prepared from stationary-phase cultures. In AP Brucella broth lacking urea, the number of both wild-type and HPKY08 (luxS ${ }^{-}$mutant) $H$. pylori decreased rapidly at $\mathrm{pH} 1$, and no cells were viable after $1 \mathrm{~h}$ (data not shown). In medium containing $30 \mathrm{mM}$ urea at $\mathrm{pH} \mathrm{1,} \mathrm{both} \mathrm{the} \mathrm{HPKY08} \mathrm{and} \mathrm{wild-type} \mathrm{strains}$ recovered to $10^{4}$ c.f.u. $\mathrm{ml}^{-1}$ at $1 \mathrm{~h}$ after inoculation of approximately $10^{6}$ c.f.u. $H$. pylori $\mathrm{ml}^{-1}$. In the presence of $30 \mathrm{mM}$ urea, both strains were maintained at $10^{4}$ c.f.u. $\mathrm{ml}^{-1}$ for $3 \mathrm{~h}$ of incubation at $\mathrm{pH}$ 3. Similar results were obtained when the strains were in exponential phase (data not shown). These results show that there are no differences in survival between the wild-type and $\operatorname{luxS} S^{-}$ mutant strains under acidic conditions in the presence of urea.

\section{Adhesion of HPKY08 H. pylori strain to MKN45 cells}

The adhesion activities of HPKY08 (luxS $S^{-}$) and wild-type H. pylori, which were obtained from 3-day cultures on BHI agar supplemented with $7 \%$ horse blood, were assessed by flow cytometry. The mean fluorescence intensities of the wild-type strain and HPKY08 (luxS $S^{-}$strains were 
$2622 \cdot 3 \pm 1400 \cdot 8$ and $1934 \cdot 7 \pm 932 \cdot 6$, respectively, at m.o.i. $=600$, and there was no significant difference in adhesion activity between the two strains in any of the experiments (m.o.i. $=60$ or 6000 ). These results indicate that mutation of the luxS gene does not affect the adherence activity of $H$. pylori to AGS cells.

\section{Colonization of Mongolian gerbils with HPKY08}

In the Mongolian gerbil experiment, the detectable frequency of HPKY08 (luxS $S^{-}$) by culture was lower than that of the wild-type strain. At 1,4 and 12 weeks after infection with TK1402, the rates of $H$. pylori-positive gerbils to total gerbils were $4 / 4,4 / 5$ and $3 / 5$, and mean colonization numbers were $10^{2 \cdot 61} \pm 10^{1 \cdot 56}, 10^{3 \cdot 70} \pm 10^{0 \cdot 91}$ and $10^{5 \cdot 05} \pm$ $10^{2 \cdot 51}$ c.f.u. (g gastric mucus) ${ }^{-1}$, respectively, upon culture using M-BHM agar plates (Fig. 5). In this culture method, the detection limit of $H$. pylori was $10^{2 \cdot 3}$ c.f.u. (g gastric mucus $)^{-1}$. However, at 1,4 and 12 weeks after infection with HPKY08, no $H$. pylori was isolated from the gastric mucus.

qRT-PCR was applied to determine the relative number of $H$. pylori in the gerbil stomach samples, inoculated with both strains (Fig. 5). At 1 week after infection with $H$. pylori, the mean relative number of the luxS mutant

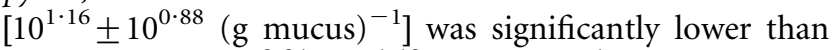

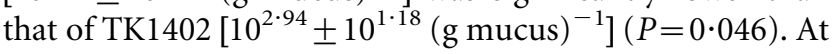
12 weeks after infection, the mean relative number of the luxS mutant was $10^{2 \cdot 24} \pm 10^{0 \cdot 28}$ (g mucus) ${ }^{-1}$, which was significantly lower than that of the wild-type strain $\left[10^{3 \cdot 86} \pm 10^{0 \cdot 43}\right.$ (g mucus) $\left.^{-1}\right](P<0 \cdot 01)$. These data indicate that the colonization rate of the luxS mutant was significantly lower than that of TK1402 in gerbil stomachs, and that the luxS gene plays an important role in $H$. pylori infectivity in gerbils.

For comparison of survival rates between the HPKY08 and TK1402 strains in the gerbil stomachs, we assessed the bacterial number in stomachs at $1 \mathrm{~h}$ after inoculation. The mean colonization number of HPKY08 $\left[10^{3 \cdot 0} \pm 10^{1 \cdot 3}\right.$ c.f.u. (g mucus $)^{-1}$ ] was not significantly different from that of TK1402 $\left.\left(10^{3 \cdot 3} \pm 10^{0 \cdot 8} \text { c.f.u. (g mucus) }\right)^{-1}\right]$. This suggests that there are no differences in survival in gerbil stomachs between the HPKY08 and TK1402 strains at this early time point.

Serum antibody titres from gerbils infected with $H$. pylori were assessed by ELISA at 12 weeks after infection. The ELISA value of serum antibodies from gerbils infected with HPKY08 $\left(A_{490} 0 \cdot 24 \pm 0 \cdot 90\right)$ was significantly lower than that of gerbils infected with TK1402 $\left(A_{490} \quad 0 \cdot 992 \pm 0 \cdot 474\right.$; $P<0 \cdot 042)$. However, the antibody titre of gerbils infected with HPKY08 was higher than that of non-infected gerbils $\left(A_{490} 0 \cdot 042 \pm 0 \cdot 043 ; P<0 \cdot 01\right)$.

On histopathological examination, no pathological changes were observed in the stomachs of gerbils infected with HPKY08 $\left(\right.$ luxS $S^{-}$) at 1 and 3 months after inoculation
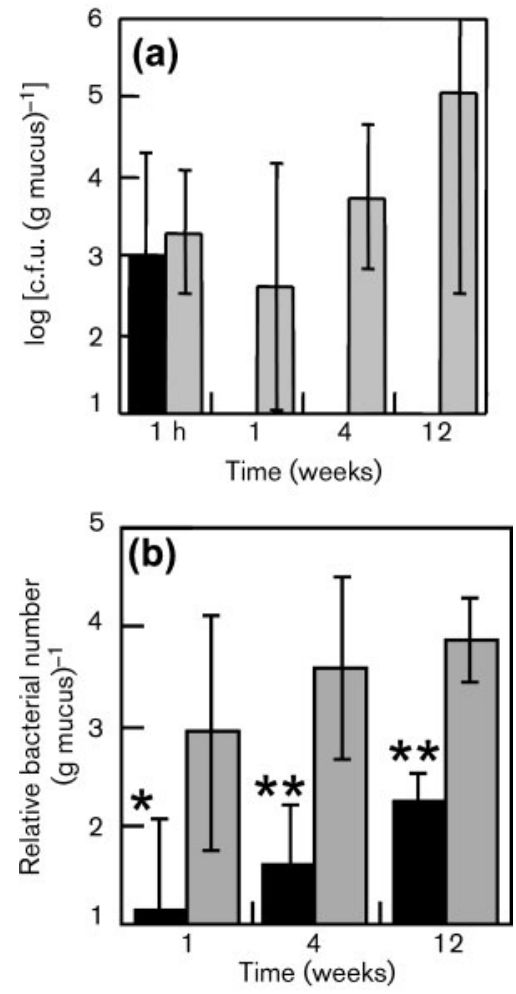

Fig. 5. Effect of luxS mutation on the infectivity of $H$. pylori to Mongolian gerbils, estimated by culture (a) and qRT-PCR (b). (a) A total of $1-2 \times 10^{9}$ c.f.u. of HPKY08 (black bars) and TK1402 (grey bars) strains were inoculated into Mongolian gerbils per os in Hanks' solution on two consecutive days. Error bars indicate standard deviation $(n=4,1 \mathrm{~h}$ and 1 week; $n=5$, 4 and 12 weeks). (b) The 16S rRNA gene of $H$. pylori (black bars, HPKY08; grey bars, TK1402) was detected in gerbil stomachs by SYBR Green real-time RT-PCR. A single PCR amplicon from cDNA prepared from total RNA extracted from stomach samples at each of the times is shown. *Significantly different from TK1402-infected gerbils $(P<0.05)$; ** significantly different from TK1402-infected gerbils $(P<0 \cdot 01)$.

(Fig. 6a). However, severe gastritis was observed in gerbil stomachs infected with TK1402 at 3 months after infection (Fig. 6b). This suggests that the infectivity of the mutant strain is insufficient for induction of pathogenic changes in the gerbil stomach.

\section{Implications of experimental data}

In Mongolian gerbil experiments, it was shown that the total bacterial number of $H$. pylori HPKY08 luxS $S^{-}$cells in the mucus of the stomach was significantly lower than that of H. pylori TK1402. H. pylori possesses several putative colonization factors, including flagella motility (Marshall \& Warren, 1984), various adhesins (Evans et al., 1993; Falk et al., 1993; O'Toole et al., 1995), vacA (Salama et al., 2001) and urease (Marshall \& Warren, 1984; Tsuda et al., 1994), some of which have been shown to be necessary for gastric 

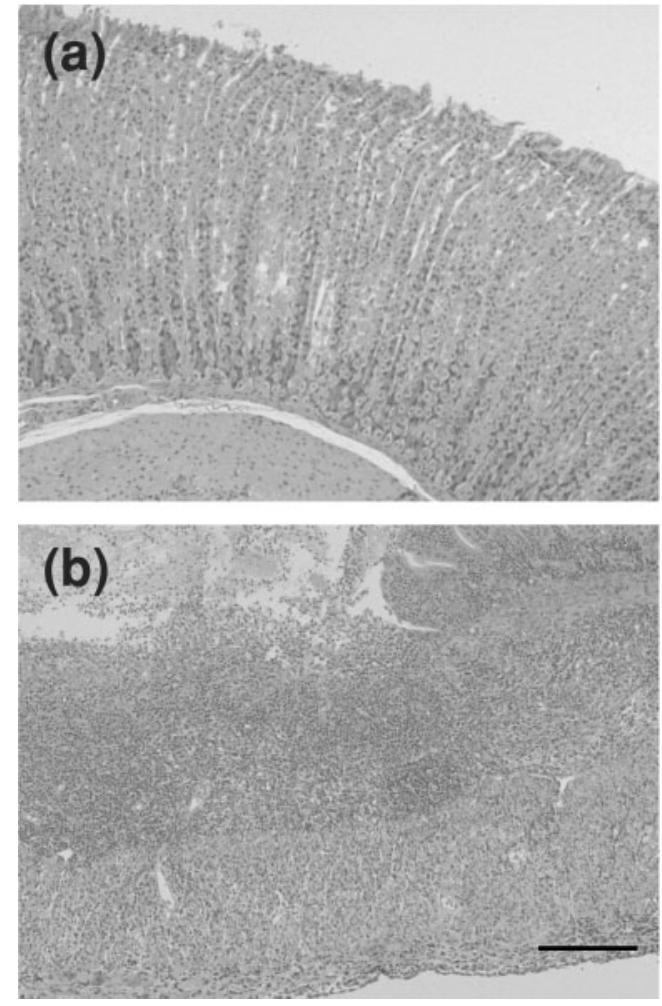

Fig. 6. Histopathological findings in gastric mucosa of Mongolian gerbils 12 weeks after $H$. pylori HPKY08 (a) and TK1402 (b) infection (haematoxylin and eosin stain). Bar, $200 \mu \mathrm{m}$.

colonization. Isogenic fla $A^{-}$, flaB ${ }^{-}$and flaA fla $B^{-}$mutant strains do not show full motility, and are unable to form effective colonies in the stomachs of germ-free piglets (Eaton et al., 1996). The experimental data indicate that both flagellin species are necessary for full colonization by H. pylori. Iwao et al. (1999) have recently reported that phenotypic variants of $H$. pylori strains lacking either motility or urease activity lose their ability to colonize. In our study, there were no differences in flagella formation between $H$. pylori HPKY08 (luxS $S^{-}$mutant) and wild-type strains. However, H. pylori HPKY08 exhibited both lower motility on soft agar medium and lower colonization potential in gerbil stomach when compared with TK1402.

In addition, it has been reported that motile strains are able to colonize regardless of their vacuolating cytotoxin activities, thus indicating that vacuolating cytotoxin is not associated with epithelial damage in the gastric mucosa (Iwao et al., 1999). In this study, it was shown that the luxS mutant had various virulence factors, such as vacuolating cytotoxin activity and hummingbird phenotype, caused by CagA protein injected into epithelial cells through type IV secretion machinery (data not shown). On histopathological examination, severe damage was observed in the gastric mucosa of gerbils infected with TK1402, but no damage was detected in the gastric mucosa of gerbils infected with
HPKY08. It is therefore possible that induction of gastric damage depends on bacterial numbers in the stomach, and that the colonization potential is not associated with expression of these virulence factors.

H. pylori possesses numerous adhesive molecules, which are important for colonization in animal models. Yamaoka et al. (2002) have reported that the switch status of several genes, such as oipA, hopZ, hopO and hopP, encoding outermembrane proteins influences the colonization of $H$. pylori in the stomachs of C57/BL mice. In their study, when two or more of these genes were 'off, colonization rates were markedly reduced compared with conditions under which all genes were 'on'. However, in this study, it was shown that the adhesion of HPKY08 cells to MKN45 cells was almost identical to that of the wild-type TK1402 strain. In addition, there was no significant difference in the bacterial number in gastric mucosa at $1 \mathrm{~h}$ after inoculation between TK1402and HPKY08-infected Mongolian gerbils. These results indicate that the decreased infectivity of the HPKY08 luxS mutant strain was not due to decreased adhesion activity.

Urease activity in $H$. pylori is required for its acid resistance, and thus for survival under the low-pH conditions of the stomach. In the present study, the urease activity of HPKY08 was as strong as that of TK1402 (data not shown). Both the wild-type TK1402 and the mutant HPKY08 strains survived at $\mathrm{pH} 3$ in the presence of $30 \mathrm{mM}$ urea. Therefore, the lower infectivity of HPKY08 is unlikely to be due to lower urease activity. In fact, there were no differences in the survival of the two strains in the stomachs of gerbils immediately after inoculation.

As shown by the qRT-PCR results (Fig. 5b) at 12 weeks after inoculation with HPKY08 $\left(l u x S^{-}\right)$, considerable amounts of mRNA for $H$. pylori $16 \mathrm{~S}$ rRNA were detected in the mucus of gerbil stomach, but isolation of $H$. pylori from gastric mucus was negative. We performed an animal experiment using the luxS-complemented strain HPKY21 in the Mongolian gerbil model. In the qRT-PCR assay, the number of colonizing $H$. pylori luxS-complemented HPKY21 strain cells was more than that of the $\operatorname{luxS} S^{-}$mutant strain. In addition, there was no significant difference in the number of colonizing $H$. pylori between TK1402- and HPKY21-infected Mongolian gerbils (data not shown). These results support a hypothesis that the luxS mutation is involved in the colonization and persistent infection of $\mathrm{H}$. pylori in the gastric mucosa of the Mongolian gerbil.

Serum antibody titres in gerbils infected with HPKY08 were slightly higher than those in non-infected gerbils. These data imply that HPKY08 colonized the gastric mucus of the gerbils, although the number of colonizing $H$. pylori cells was below the detection limit, $2 \times 10^{2}$ c.f.u. (g mucus) ${ }^{-1}$, for the culture-based assay. In gerbil stomachs, numerous other types of bacteria form colonies, and some of these may possess the luxS/AI-2 system. It is possible that the AI-2 produced by these bacteria affected the luxS mutant strain, thus allowing the mutant to survive in the gerbil stomach. 
The biosynthetic pathway of AI-2 has recently been clarified (Schauder et al., 2001). AI-2 is generated from $S$-adenosylmethionine (SAM) in three enzymic steps. SAM is an essential metabolite used in central metabolism. Following methyl transfer from SAM to its various substrates, $S$-adenosylhomocysteine (SAH) is formed. SAH is a potent inhibitor of SAM-dependent methyl transferase, and thus must be eliminated. However, the luxS mutant is unable to convert the second product, $S$-ribosylhomocysteine (SRH), which is the substrate of LuxS, to AI-2 and homocysteine (third product). The accumulation of SAH in the mutant may inhibit bacterial cell growth. In fact, the viable cell number of HPKY08 in the stationary phase was lower than that of TK1402.

\section{ACKNOWLEDGEMENTS}

This work was supported by Grants for Scientific Research (\#16590371 and \#17590399) from the Ministry of Education, Culture, Sport, Science and Technology, Japan.

\section{REFERENCES}

Balestrino, D., Haagensen, J. A., Rich, C. \& Forestier, C. (2005). Characterization of type 2 quorum sensing in Klebsiella pneumoniae and relationship with biofilm formation. J Bacteriol 187, 2870-2880.

Blaser, M. J. (1992). Helicobacter pylori: its role in disease. Clin Infect Dis 15, 386-391.

Blehert, D. S., Palmer, R. J., Jr, Xavier, J. B., Almeida, J. S. \& Kolenbrander, P. E. (2003). Autoinducer 2 production by Streptococcus gordonii DL1 and the biofilm phenotype of a luxS mutant are influenced by nutritional conditions. J Bacteriol 185, $4851-4860$

Chen, X., Schauder, S., Potier, N., Van Dorsselaer, A., Pelczer, I., Bassler, B. L. \& Hughson, F. M. (2002). Structural identification of a bacterial quorum-sensing signal containing boron. Nature 415, 545-549.

Drazek, E. S., Dubois, A. \& Holmes, R. K. (1994). Characterization and presumptive identification of Helicobacter pylori isolates from rhesus monkeys. J Clin Microbiol 32, 1799-1804.

Eaton, K. A., Suerbaum, S., Josenhans, C. \& Krakowka, S. (1996). Colonization of gnotobiotic piglets by Helicobacter pylori deficient in two flagellin genes. Infect Immun 64, 2445-2448.

Eaton, K. A., Cover, T. L., Tummuru, M. K. R., Blaser, M. J. \& Krakowka, S. (1997). Role of vacuolating cytotoxin in gastritis due to Helicobacter pylori in gnotobiotic piglets. Infect Immun 65, 3462-3464.

Evans, D. G., Karjalainen, T. K., Evans, D. J., Graham, D. Y. \& Lee, C. H. (1993). Cloning, nucleotide sequence, and expression of a gene encoding an adhesin subunit protein of Helicobacter pylori. J Bacteriol 175, 674-683.

Falk, P., Roth, K. A., Boren, T., Westblom, T. U., Gordon, J. I. \& Normark, S. (1993). An in vitro adherence assay reveals that Helicobacter pylori exhibits cell lineage-specific tropism in the human gastric epithelium. Proc Natl Acad Sci U S A 90, 2035-2039.

Fong, K. P., Chung, W. O., Lamont, R. J. \& Demuth, D. R. (2001). Intra- and interspecies regulation of gene expression by Actinobacillus actinomycetemcomitans LuxS. Infect Immun 69, 7625-7634.
Forsyth, M. H. \& Cover, T. L. (2000). Intercellular communication in Helicobacter pylori: luxS is essential for the production of an extracellular signaling molecule. Infect Immun 68, 3193-3199.

Fujioka, T., Kodama, R., Honda, S., Guei-Hua, G., Nishizono, A. \& Nasu, M. (1997). Long-term sequelae of experimental gastritis with Helicobacter pylori: a 5-year follow-up study. J Clin Gastroenterol 25 (Suppl. 1), S8-S12.

Fuqua, W. C., Winans, S. C. \& Greenberg, E. P. (1994). Quorum sensing in bacteria: the LuxR-LuxI family of cell density-responsive transcriptional regulators. J Bacteriol 176, 269-275.

Graham, D. Y. (1989). Campylobacter pylori and peptic ulcer disease. Gastroenterology 96, 615-625.

Hardie, K. R., Cooksley, C., Green, A. D. \& Winzer, K. (2003). Autoinducer 2 activity in Escherichia coli culture supernatants can be actively reduced despite maintenance of an active synthase. Microbiology 149, 715-728.

Hirayama, F., Takagi, S., Yokoyama, Y., Iwao, E. \& Ikeda, Y. (1996). Establishment of gastric Helicobacter pylori infection in Mongolian gerbils. J Gastroenterol 31, 24-28.

Iwao, E., Hirayama, F., Takagi, S., Yokoyama, Y. \& Ikeda, Y. (1999). Virulence factors of Helicobacter pylori affecting its gastric colonization in Mongolian gerbils. J Gastroenterol 34 (Suppl. 11), 47-54.

Jeon, B., Itoh, K., Misawa, N. \& Ryu, S. (2003). Effects of quorum sensing on flaA transcription and autoagglutination in Campylobacter jejuni. Microbiol Immunol 47, 833-839.

Joyce, E. A., Bassler, B. L. \& Wright, A. (2000). Evidence for a signaling system in Helicobacter pylori: detection of a luxS-encoded autoinducer. J Bacteriol 182, 3638-3643.

Kim, S. Y., Lee, S. E., Kim, Y. R., Kim, C. M., Ryu, P. Y., Choy, H. E., Chung, S. S. \& Rhee, J. H. (2003). Regulation of Vibrio vulnificus virulence by the LuxS quorum-sensing system. Mol Microbiol 48, $1647-1664$.

Lee, A., O'Rourke, J., Ungria, M. C. D., Robertson, B., Daskalopoulos, G. \& Dixon, M. F. (1997). A standardized mouse model of Helicobacter pylori infection: introducing the Sydney strain. Gastroentero$\log y 112,1386-1397$.

Loh, J. T., Forsyth, M. H. \& Cover, T. L. (2004). Growth phase regulation of flaA expression in Helicobacter pylori is luxS dependent. Infect Immun 72, 5506-5510.

Lyon, W. R., Madden, J. C., Levin, J. C., Stein, J. L. \& Caparon, M. G. (2001). Mutation of $l u x S$ affects growth and virulence factor expression in Streptococcus pyogenes. Mol Microbiol 42, 145-157.

Marshall, B. J. \& Warren, J. R. (1984). Unidentified curved bacilli in the stomach of patients with gastritis and peptic ulceration. Lancet $\mathbf{I}$, 1311-1315.

Matsumoto, S., Washizuka, Y., Matsumoto, Y., Tawara, S., Ikeda, F., Yokota, Y. \& Karita, M. (1997). Induction of ulceration and severe gastritis in Mongolian gerbil by Helicobacter pylori infection. J Med Microbiol 46, 391-397.

Nakagawa, S., Osaki, T., Fujioka, Y., Yamaguchi, H. \& Kamiya, S. (2005). Long-term infection of Mongolian gerbils with Helicobacter pylori: microbiological, histopathological, and serological analyses. Clin Diagn Lab Immunol 12, 347-353.

Ohtani, K., Hayashi, H. \& Shimizu, T. (2002). The luxS gene is involved in cell-cell signalling for toxin production in Clostridium perfringens. Mol Microbiol 44, 171-179.

Osaki, T., Taguchi, H., Yamaguchi, H., Kumada, J., Ogata, S. \& Kamiya, S. (1997). Studies on the relationship between adhesive activity and haemagglutination by Helicobacter pylori. J Med Microbiol 46, 117-121. Osaki, T., Taguchi, H., Yamaguchi, H. \& Kamiya, S. (1998). Detection of Helicobacter pylori in fecal samples of gnotobiotic 
mice infected with $H$. pylori by an immunomagnetic-bead separation technique. J Clin Microbiol 36, 321-323.

O’Toole, P. W., Janzon, L., Doig, P., Huang, J. Z., Kostrzynska, M. \& Trust, T. J. (1995). The putative neuraminyllactose-binding hemagglutinin HpaA of Helicobacter pylori CCUG 17874 is a lipoprotein. J Bacteriol 177, 6049-6057.

Parsonnet, J., Friedman, G. D., Vandersteen, D. P., Chang, Y., Vogelman, J. H. \& Orentreich, N. (1991). Helicobacter pylori infection and the risk of gastric carcinoma. N Engl J Med 325, 1127-1131.

Salama, N. R., Otto, G., Tompkins, L. \& Falkow, S. (2001). Vacuolating cytotoxin of Helicobacter pylori plays a role during colonization in a mouse model of infection. Infect Immun 69, 730-736.

Schauder, S., Shokat, K., Surette, M. G. \& Bassler, B. L. (2001). The LuxS family of bacterial autoinducers: biosynthesis of a novel quorum-sensing signal molecule. Mol Microbiol 41, 463-476.

Schneider, R., Lockatell, C. V., Johnson, D. \& Belas, R. (2002). Detection and mutation of a luxS-encoded autoinducer in Proteus mirabilis. Microbiology 148, 773-782.

Sircili, M. P., Walters, M., Trabulsi, L. R. \& Sperandio, V. (2004). Modulation of enteropathogenic Escherichia coli virulence by quorum sensing. Infect Immun 72, 2329-2337.

Sperandio, V., Mellies, J. L., Nguyen, W., Shin, S. \& Kaper, J. B. (1999). Quorum sensing controls expression of the type III secretion gene transcription and protein secretion in enterohemorrhagic and enteropathogenic Escherichia coli. Proc Natl Acad Sci U S A 96, 15196-15201.

Stroeher, U. H., Paton, A. W., Ogunniyi, A. D. \& Paton, J. C. (2003). Mutation of luxS of Streptococcus pneumoniae affects virulence in a mouse model. Infect Immun 71, 3206-3212.
Surette, M. G. \& Bassler, B. L. (1999). Regulation of autoinducer production in Salmonella typhimurium. Mol Microbiol 31, 585-595.

Thompson, L. J., Merrell, D. S., Neilan, B. A., Mitchell, H., Lee, A. \& Falkow, S. (2003). Gene expression profiling of Helicobacter pylori reveals a growth-phase-dependent switch in virulence gene expression. Infect Immun 71, 2643-2655.

Tsuda, M., Karita, M., Morshed, M. G., Okita, K. \& Nakazawa, T. (1994). A urease-negative mutant of Helicobacter pylori constructed by allelic exchange mutagenesis lacks the ability to colonize the nude mouse stomach. Infect Immun 62, 3586-3589.

Wen, Z. T. \& Burne, R. A. (2004). LuxS-mediated signaling in Streptococcus mutans is involved in regulation of acid and oxidative stress tolerance and biofilm formation. J Bacteriol 186, 2682-2691.

Wotherspoon, A. C., Doglioni, C., Diss, T. C., Pan, L., Moschini, A., De Boni, M. \& Isaacson, P. G. (1993). Regression of primary lowgrade B-cell gastric lymphoma of mucosa-associated lymphoid tissue type after eradication of Helicobacter pylori. Lancet 342, 575-577.

Yamaguchi, H., Osaki, T., Kai, M., Taguchi, H. \& Kamiya, S. (2000). Immune response against a cross-reactive epitope on the heat shock protein 60 homologue of Helicobacter pylori. Infect Immun $\mathbf{6 8}$, 3448-3454.

Yamaoka, Y., Kita, M., Kodama, T., Imamura, S., Ohno, T., Sawai, N., Ishimaru, A., Imanishi, J. \& Graham, D. Y. (2002). Helicobacter pylori infection in mice: role of outer membrane proteins in colonization and inflammation. Gastroenterology 123, 1992-2004.

Yokota, K., Kurebayashi, Y., Takayama, Y. \& 7 other authors (1991). Colonization of Helicobacter pylori in the gastric mucosa of Mongolian gerbils. Microbiol Immunol 35, 475-480. 